\title{
Reconstruction of Bone Defects after Tumor Resection Using Present Methods (Mini-Review). Bone Reconstruction Algorithm
}

\section{Evgeniya Anastasieva* and IA Kirilova}

Novosibirsk Research Institute of Traumatology and Orthopedics n.a. Ya.L. Tsivyan, Novosibirsk, Russian Federation

*Corresponding Author: Evgeniya Anastasieva, Ph.D. Candidate, Novosibirsk Research Institute of Traumatology and Orthopedics n.a. Ya.L. Tsivyan, Novosibirsk, Russian Federation.

DOI: $10.31080 /$ ASOR.2020.03.0186

\section{Abstract}

The replacement of large bone defects after segmental bone resections in patients with bone tumors is an actual problem in orthopedics. The main difficulty it causes especially in cases of disturbance of normal biomechanics. Especially when the "gold standard" of reconstruction with bone autograft is not possible, because of the defect, which can be so extensive that any autograft stock will be not enough. Therefore, allografts based on demineralized bone with optimal properties for osteoregeneration can become the necessary alternative for bone replacement. Nowadays composite materials is possible to be programmed to have the specific properties by changing its compound. After concluding the results of the literature analysis, we revealed that the allograft in combination with additional components is comparable by its effectiveness with autograft.

As an additional component to improve osteoregeneration mesenchymal stem cells of both bone marrow and adipose tissue can be used. However, it is noteworthy that the analyzed studies did not reveal the influence of stem cells on the tumor recurrence. So, the authors tell about necessity of further researches. Some authors still prefer traditional methods of bone traction despite obtaining own satisfactory results of defects reconstruction with allografts. Such opinion is based on proven effectiveness of the method, structural stability of construction during treatment period and ability to adjust the process of bone regeneration at any stage.

Our goal was to analyze publications over the recent 10 years, which report about results of experiments and clinical studies with performing massive bone reconstruction after bone tumor resection using auto- and allografts, or other technique. Based on the literature analysis we have updated our previous algorithm for graft selection in replacement of large bone defects after segmental bone resections.

Keywords: Bone Tumors; Osteooncology; Orthopedics; Bone Defect; Bone Reconstruction; Bone Resection; Bone Autograft; Bone Allograft

\section{Introduction}

Large bone defects replacement after segmental bone resections in patients with bone tumors remains an actual problem for orthopedics. It is known that an autograft is the most acceptable material for reconstructive surgery, but its usage is limited [1]. Every graft should have certain properties - to have greater weight bearing resistance, modeled for a defect to be filled. So here is the problem - when the defect is much more, then it can be reconstructed by autograft, when normal biomechanics corrupted and structural stability of the whole bone may be corrupted $[2,3]$.

\section{Materials and Method}

Writing our review, we searched for significant study results for past 10 years.

\section{Results of the Review}

When replacing such defects, it is preferred using an allograft, despite the fact that compared to an autograft there will be fewer regeneration mechanisms involved. Also here is the need for vascularization for the entire transplant, since bone restoration does not occur due to the lack of adequate microcirculation. Thus, the size of the implant and its vascularization are crucial for tissue viability, nearest tendons and nerve functions $[2,4,5]$. The sympathetic nervous system importantly affects the regeneration of bone tissue, determining the density of future bone, the speed of reparative processes, the activity of osteoblasts and osteoclasts [4]. This effect is partially explained by the hypothesis of the presence of $\beta 2$ adrenergic receptors in bone tissue cells [4]. 
Another important graft property - its stability. Since stability is one to determine the frequency of infectious complications occurrence, the speed of tissue reparative processes. In the case of instability of the whole structure the irritation of the surrounding tissues occurs. This is able to cause an inflammatory process, pain syndrome and other clinical syndromes [6].

When relacing bone defects, the following oncological aspects should be taken into account:

1. Benignancy or malignancy of resected tissue: This aspect determines the surgical method of affected area resection (adherence with ablasticity standards) and further medical support during the postoperative period [7-19].

1. Age: The regenerative potential of bone tissue in children is much higher than in adults, which makes it possible to restore the affected bone as physiologically as possible. Children require a more delicate approach, since surgical intervention should ensure the preservation of the function and stability of the affected segment according the dynamics of growth [20$24]$.

1. Process localization and its type (primary or metastatic): This aspect affects the volume of the resected area. Postoperative chemotherapy support is selected individually [25-29].

1. In elderly with metastatic processes, surgery is often a palliative measure, and surgical tactics are adjusted accordingly [30-33].

1. In case of repeated surgical interventions at the same bone segment, in addition with the ablastic principles, which determine the volume of the resected area, it is extremely important for the regeneration of the removed bone fragment to maintain the stability of the chosen construction [32,34].

The relevance of our literature review is due to the complex graft rearrangement. Because in case of the huge defect, of more than $4 \mathrm{~cm}^{3}$, the microvascular reconstruction and connected further regeneration of bone tissue are complicated [35].

According to the literature data large bone tissue defects can be divided into two groups:

- Defects in the bone structure, which are not affecting the biomechanics of the limb, but not allowing to perform osteosynthesis or arthroplasty;
- Segmental bone defects that affect normal biomechanics and structural stability of the bone as an organ.

This division of defects allows to create an algorithm for selecting a better tactic for treatment. As an alternative, instead of using autograft, allograft can be installed. Allograft of bone tissue is based on demineralized allograft bone, which possess the necessary osteoinductive properties $[35,36]$.

Some composite materials used for bone replacement have additional effects due to the addition of plasticizers giving elastic properties, various drugs or even stem cells. The effectiveness of an allograft in combination with described additional components is comparable by its effectiveness with the autograft. Also effects of such composition can be seen during long-term postoperative period $[37,38]$.

M. Gharedaghi., et al. studies revealed that the results when using allograft to replace large bone defects after resection either benign or malignant bone tumors almost do not differ the results when using autologous bone [37]. The number of other authors came to similar conclusion in their researches [33,39-48].

According to the literature data, 5-year survival rate is $73 \%$ and above, tumor recurrency rate is less than $13 \%$, and the authors do not associate these cases with the allograft usage $[39,43,48]$. Rather, they are caused by technique errors during the surgical intervention. Satisfactory results obtained using allograft in orthopedic oncology in pediatric patients have been reported. Meanwhile the case of massive resorption of the entire transplant is described $[41,45,49]$.

When replacing the weight-bearing part of the bone with an allograft, transplant revealed not being strong enough, and its 5 to 10 years survival rate varies in range from $75 \%$ to $83 \%$ [39]. In cases with severe bone damage involving the joint surfaces there is an indications for total bone resection with total arthroplasty [6,50-57]. This decision due to strong ablastic protocol proved by international oncoorthopedics guide. D.A. Müller., et al. in such cases proposed to use bone allograft in addition. Authors explain that additional graft makes up for the lost volume of bone tissue [53], while the autograft takes weight-bearing function. The evidence of the effectiveness of D.A. Müller., et al. technique proved by other authors. Thus, K. Nakamura reported about total arthroplasty in combination with bone allograft implantation, which allowed to obtain good functional results [58]. Some authors tell about the advantages of the combined bone auto- and allograft usage substantiating its positive results by the fact that the autograft already had osteogenic and MSC cells in its structure providing the stimulation effect for bone regeneration $[47,59,60]$. 
R. Gouron in his study showed that pediatric patients require a special delicate approach. In cases with those young patients the indications for certain transplant usage, should be clearly defined [49]. Moreover, in treating major bone defects, especially when the area is exposed to a high weight load, it is important to use a technique with a supporting and delimiting structure like a membrane. This approach allows us to sculpt the shape of the future bone regenerate. Analyzing the results of other studies, R. Gouron noted, that the postoperative complications like bone defect consolidation failure were revealed in $35 \%$ of patients, but all of them were corrected at further period. Among pediatric patients, the author described a single case of the total allograft resorption [41].

RA Glennie., et al. reported about usage potential of allogeneic material in surgery right after single-unit resection of a spinal tumor [61]. When more than one level defect reconstructing, the authors recommend the using of bone allograft or its combination with a cage, which is a metal hollow frame insert between the vertebral bodies.

It is promising to implant the combined elastic allogeneic material with the necessary chemotherapy, which provides controlled desorption to confirm the effective drug presence for a certain period.

The use of stem cells as an additional component for improving osteoregeneration was reported by P. Avril., et al [59]. The results of their study showed the result using bone marrow MSCs, it had no bone tumor relapse consequences. However, they reported that existing lung metastases able to progress. When using adipose tissue stem cells no relapse was observed either. There no effect of MSCs on tumor cells was detected in vivo. However, the authors talk about the need of further research to confirm received results.

In cases when unable to use allograft of autograft, some authors prefer the hardware extension of the limb. The main positive factors of this method are its time-proven effectiveness, stability and adjustable process of bone regeneration [62,63]. M.P. Bus., et al. also prefer Osteosynthesis by metal constructions in spite of their own satisfactory results of using allograft. They explaining this by the complication rate increasing when using allograft bone defect replacement in a volume of more than $15 \mathrm{~cm}^{3}$ [64].

In connection with the increasing allograft usage, it became necessary to think about the need to create a virtual 3D allograft bank. This would allow accurate selection of material, taking into account the available research results, such as CT and MRI, to replace major bone defects in orthopedic oncology $[65,66]$. There is also a tendency to fill such defects with 3D printed materials containing a combination of bone substitute substance and allograft [67].

Author's algorithm for bone defects replacement

Based on the literature data, we developed an algorithm for selecting an appropriate transplant to replace large bone defects after segmental bone resection. This algorithm can serve as the basis for a differentiated treatment approach. It may also be useful in the treatment of patients with cancer, with the additional implementation of appropriate antineoplastic measures.

We propose to divide all bone defects based on possibility of using bone autograft to replace a bone defect. The next criteria helping to choose a transplant is the type of bone defect, effect on the biomechanics and structural stability of the bone. Adopting the whole scheme according to the possible need for additional external bone fixation. If it is impossible to fill a large bone defect an alternative methods are accepted: installation of a titanium nickelide implant, usage of Ilizarov hardware. However, the surgeon decides individually the issue of additional external fixation of any defect (outside the proposed tactics) depending on the stability of the bone damage. Also, for each patient, the question of chemotherapeutic support should be solved individually.

The diagram is built from left to right from the most preferred treatment option (Figure 1).

When choosing treatment tactics, first of all, we suggest to determ whether the size of the defect and its shape allow the use of an autograft. The most preferred option is to use patient's own tissue to fill the bone defect. In the absence of autologous bone material at the required quantity, the allo-/autograft combination or allograft only is acceptable. If at the step of size and shape determining it becomes clear that autologous material use only is impossible, then we need to estimate if the bone defect affect the biomechanics.

In the case of a biomechanically insignificant bone defect, the question of the need for additional supporting structures should be solved. Then it is needed to decide by which material cage should be filled: combination of auto- and/or allograft in accordance with existing treatment methods.

\section{Conclusion}

In the presence of a biomechanically significant defect, the choice of the method of its replacement depends on the size of the defect. Replacing a defect with a less than $15 \mathrm{~cm}^{3}$ volume can be 


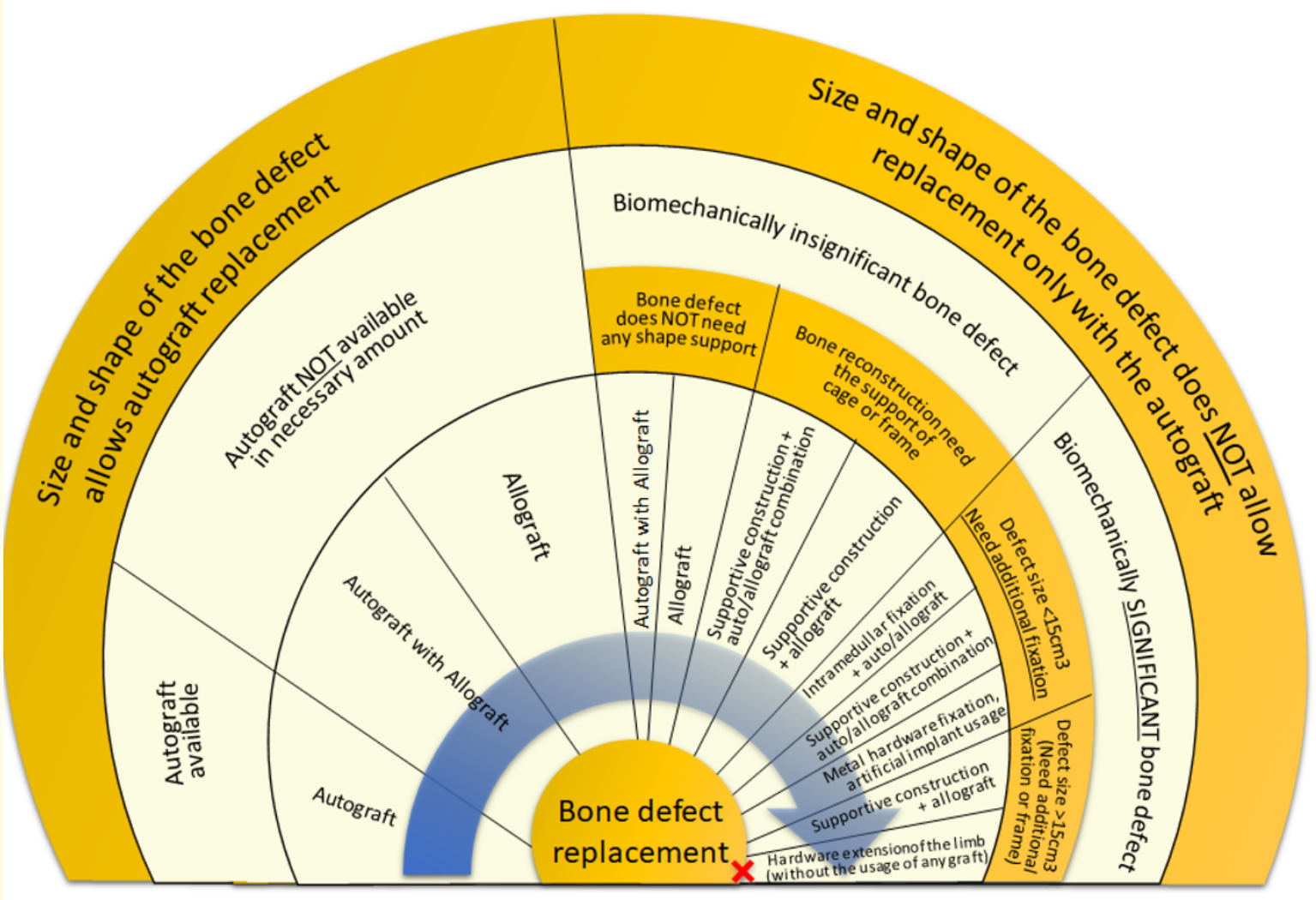

Figure 1: Author's algorithm for bone defects replacement (2017 update).

performed by various methods: intramedullary fixation with an auto and allograft combination; bone restoration using a supporting cage with a combination of auto- and allograft; defect fixation using an artificial implant (for example, titanium nickelide). However, with a defect with a volume of more than $15 \mathrm{~cm}^{3}$, it is preferred to choose hardware traction of the affected segment without the usage of any implants.

\section{Conflict of Interest}

The authors had no financial interest or any conflict of interest exists.

\section{Bibliography}

1. Qu H., et al. "Reconstruction of segmental bone defect of long bones after tumor resection by devitalized tumor-bearing bone". World Journal of Surgical Oncology 13 (2015): 282.

2. Guerado E and Caso E. "Challenges of bone tissue engineering in ortho paedic patients". World Journal of Orthopedics 8.2 (2017): 87-98.

3. Glatt V., et al. "A Concert between biology and biomechanics: the influence of the mechanical environment on bone healing". Frontiers in Physiology 7 (2016): 678.
4. Elefteriou F., et al. "Control of Bone Remodeling by the Peripheral Sympathetic Nervous System". Calcified Tissue International 94.1 (2014): 140-151.

5. Muramatsu K., et al. "Stimulation of neo-angiogenesis by combined use of irradiated and vascularized living bone graft for oncological reconstruction". Surgical Oncology 21.3 (2012): 223-229.

6. Kazarezov MV., et al. " [Orthopedics and reconstructive surgery]”. Novosibirsk: NNIITO, NGO “BREEZE” (2008): 448.

7. Balberkin AV and Shavyrin DA. "[Clinic, diagnostics and surgical treatment of bone tumors of the knee joint region (literature review)]". Sarkomy Kostei, Myagkikh Tkanei I Opukholi Kozhi Sarcomas of Bone, Soft Tissue and Skin Tumors 1 (2013): 15-23.

8. Terskov AJu., et al. " [Our tactics in diagnostics and treatment of patients with giant cell tumor of bone]". Orthopedic Genius 2 (2013): 67-71. 
9. Dittrich C., et al. "ESMO/ASCO Recommendations for a Global Curriculum in Medical Oncology”. ESMO Open 1.5 (2016): e000097.

10. Fritzsche H., et al. " [Benign tumours and tumour-like lesions of the bone: general treatment principles]". Orthopade 46.6 (2017): 484-497.

11. Hillmann A and Gösling T. "[Benign bone tumors. General principles]”. Unfallchirurg 117.10 (2014): 873-882.

12. Leavey PJ. "Important principles in Ewing sarcoma treatment". Pediatric Blood Cancer 61.12 (2014): 2149-2150.

13. Mavrogenis AF., et al. "State-of-the-art approach for bone sarcomas". European Journal of Orthopaedic Surgery and Traumatology 25.1 (2015): 5-15.

14. Panagopoulos GN., et al. "Intercalary reconstructions after bone tumor resections: a review of treatments". European Journal of Orthopaedic Surgery and Traumatology 27.6 (2017): 737-746.

15. Borzunov DY., et al. "Reconstruction by bone transport after resection of benign tumors of tibia: A retrospective study of 38 patients". The Indian Journal of Orthopaedics 49.5 (2015): 516-522.

16. Puri A., et al. "Reconstruction of the elbow and forearm for Ewing sarcoma of ulna: A new biological technique". International Journal of Shoulder Surgery 10.2 (2016): 85-88.

17. Puri A. "Limb salvage in musculoskeletal oncology: Recent advances". Indian Journal of Plastic Surgery 47.2 (2014): 175184.

18. Puri A and Gulia A. "Management of extremity soft tissue sarcomas". The Indian Journal of Orthopaedics 45 (2011): 301306.

19. Tikhilov RM., et al. " [Surgical treatment for giant cell tumor of the bones]". Herzen Journal of Oncology 6.1 (2017): 5-11.

20. Ramphal R., et al. "Adolescent and young adult cancer: principles of care". Current Oncology 23.3 (2016): 204-209.

21. Sadovoj MA., et al. " [Surgical treatment of bone tumors in children]”. Travmatologiya Zhene Ortopediya 2 (2007): 134-137.

22. Nisichenko DV., et al. " [Subtotal resection of the tibia at the Research Institute of Pediatric Oncology and Hematology FGBI "RCRC. n.a. N.N. Blokhin" of the Ministry of Health of Russia]". Sarkomy Kostei, Myagkikh Tkanei I Opukholi Kozhi 1 (2017): 52-58.
23. Nieminen J., et al. "Orthopaedic reconstruction of complex pelvic bone defects. Evaluation of various treatment methods". Scandinavian Journal of Surgery 102.1 (2013): 36-41.

24. Ryzhikov DV., et al. "Repair of defects and osteosynthesis of long bones in children with osteoclastoma". AIP Conference Proceedings 1760.1 (2016): 020059.

25. Ivanova MV., et al. " [Endoprosthetics of the shoulder joint with a metastatic lesion of the proximal humerus on the background of immunosuppressive therapy]". Issledovaniya I Praktika V Meditsine 4.1 (2017): 58-62.

26. Khan Z., et al. "Tumours of the foot and ankle". Foot 25.3 (2015): 164-172.

27. Gupta S.K., et al. "Principles of rotationplasty". The Journal of the American Academy of Orthopaedic Surgeons 20.10 (2012): 657-667.

28. Malviya A and Gerrand C. "Evidence for orthopaedic surgery in the treatment of metastatic bone disease of the extremities: a review article". Palliative Medicine 26.6 (2012): 788-796.

29. Piccioli A., et al. "Bone metastases of unknown origin: epidemiology and principles of management". Journal of Orthopaedics and Traumatology 16.2 (2015): 81-86.

30. Cheng D., et al. "Efficacy of limb salvage with primary tumor resection simultaneously for solitary bone metastasis in limbs". World Journal of Surgical Oncology 14.1 (2015): 31.

31. Gösling T and Becker-Schiebe M. "[Surgical treatment of skeletal metastases]". Unfallchirurg 118.4 (2015): 347-363.

32. Scolaro JA and Lackman RD. "Surgical management of metastatic long bone fractures: principles and techniques". The Journal of the American Academy of Orthopaedic Surgeons 22.2 (2014): 90-100.

33. Wessling M., et al. " [Basic principles of surgical treatment of bone metastases]". Zeitschrift fur Orthopadie und Unfallchirurgie 151.3 (2013): 303-314.

34. Aponte-Tinao L.A., et al. "The principles and applications of fresh frozen allografts to bone and joint reconstruction". Orthopedic Clinics of North America 45.2 (2014): 257-269.

35. Kirilova IA., et al. " [Comparative Characteristics of Materials for Bone Grafting: Composition and Properties]. Khirurgiya Pozvonochnika 3 (2012):72-83. 
36. Podorozhnaya V.T., et al. "[Allogeneic bone materials: structure, properties, application]". Izvestiya Vysshikh Uchebnykh Zavedenii Fizika 56.12-3 (2013):14-20.

37. Gharedaghi M., et al. "Evaluation of Clinical Results and Complications of Structural Allograft Reconstruction after Bone Tumor Surgery". The Archives of Bone and Joint Surgery 4.3 (2016): 236-242.

38. Zamiri B., et al. "Reconstruction of human mandibular continuity defects with allogenic scaffold and autologous marrow mesenchymal stem cells". Journal of Craniofacial Surgery 24.4 (2013): 1292-1297.

39. Ayerza M.A., et al. "Structural allograft reconstruction of the foot and ankle after tumor resections". Journal of Musculoskeletal Surgery 100.2 (2016):149-156.

40. Chen W.M., et al. "High-grade osteosarcoma treated with hemicortical resection and biological reconstruction". Journal of Surgical Oncology 105.8 (2012): 825-829.

41. Gouron R. "Surgical technique and indications of the induced membrane procedure in children". Orthopaedics and Traumatology Surgery and Research Journal 102.1 (2016): S133-S139.

42. Halim A.S., et al. "Long-term outcome of free fibula osteocutaneous flap and massive allograft in the reconstruction of long bone defect". Journal of Plastic Reconstructive and Aesthetic Surgery 68.12 (2015): 1755-1762.

43. Han G., et al. "Reconstruction using massive allografts after resection of extremity osteosarcomas the study design: A retrospective cohort study". International Journal of Surgery 21 (2015): 108-111.

44. Houdek M.T., et al. "What Is the Outcome of Allograft and Intramedullary Free Fibula (Capanna Technique) in Pediatric and Adolescent Patients with Bone Tumors?" Clinical Orthopaedics and Related Research 474.3 (2016): 660-668.

45. Li J., et al. "Image navigation assisted joint saving surgery for treatment of bone sarcoma around knee in skeletally immature patients". Surgical Oncology 23.3 (2014): 132-139.

46. Farfalli GL., et al. "Clinical and functional outcomes of tibial intercalary allografts after tumor resection". Orthopedics 35.3 (2012): e391-e396.

47. Nogueira Drumond JM. "Benign bone tumors and tumorlike bone lesions: treatment update and new trends". Revista Brasileira de Ortopedia 44.5 (2015): 386-390.
48. Wang W., et al. "Allogeneic bone transplantation for pelvic reconstruction of large skeletal defects after tumor resection". Zhongguo Xiu Fu Chong Jian Wai Ke Za Zhi 28.3 (2014): 331334.

49. Gouron R., et al. "Bone defect reconstruction in children using the induced membrane technique: a series of 14 cases". Orthopaedics and Traumatology: Surgery and Research 99.7 (2013): 837-843.

50. King J.J., et al. "Allograft-prosthetic composite reverse total shoulder arthroplasty for reconstruction of proximal humerus tumor resections". Journal of Shoulder and Elbow Surgery 25.1 (2016): 45-54.

51. Mikailov IM., et al. " [Results of the proximal humerus endoprosthetic reconstruction after tumour resection]. Travmatologiya I Ortopediya Rossii 4 (2014): 27-35.

52. Tikhilov R.M., et al. " [Shortand middle-term results of hip arthroplasty for tumors of the proximal femur]". Travmatologiya I Ortopediya Rossii 2 (2014): 14-21.

53. Müller D.A., et al. "Allograft-prosthetic composite versus megaprosthesis in the proximal tibia. What works best?" Injury 47.4 (2016): S124-S130.

54. Al Gheshyan F., et al. "Comparison of gait parameters in distal femoral replacement using a metallic endoprosthesis versus allograft reconstruction". Journal of Orthopaedics 12.1 (2015): S25-S30.

55. Kotela A., et al. "Revision knee arthroplasty in patients with inherited bleeding disorders: a single-center experience". Medical Science Monitor 23 (2017): 129-137.

56. Rudert M., et al. "Impaction bone grafting for the reconstruction of large bone defects in revision knee arthroplasty". Operative Orthopädie and Traumatologie 27.1 (2015): 35-46.

57. Sakellariou VI and Babis GC. "Management bone loss of the proximal femur in revision hip arthroplasty: Update on reconstructive options". World Journal of Orthopedics 5.5 (2014): 614-622.

58. Nakamura K., et al. "Tumor-induced osteomalacia caused by a massive phosphaturic mesenchymal tumor of the acetabulum: A case report". Modern Rheumatology (2016): 1-5.

59. Avril P., et al. "Mesenchymal stem cells increase proliferation but do not change quiescent state of osteosarcoma cells: Potential implications according to the tumor resection status". Journal of Bone Oncology 5.1 (2016): 5-14. 
60. Kong Y., et al. "The bone marrow microenvironment is similarly impaired in allogeneic hematopoietic stem cell transplantation patients with early and late poor graft function". Bone Marrow Transplant 51.2 (2016): 249-255.

61. Glennie RA., et al. "A systematic review with consensus expert opinion of best reconstructive techniques after osseous en bloc spinal column tumor resection". Spine 41.20 (2016): S205-S211.

62. Balaev PI and Borzunov DJu. "[Possibilities of bone reconstruction according to G.A. Illizarov in the recovery treatment of patients with primary tumors of the lower leg]". Sibirskii Onkologicheskii Zhurnal 1.55 (2013): 59-64.

63. Khira YM and Badawy HA. "Pedicled vascularized fibular graft with Ilizarov external fixator for reconstructing a large bone defect of the tibia after tumor resection". Journal of Orthopaedics and Traumatology 14.2 (2013): 91-100.

64. Bus MP., et al. "Intercalary allograft reconstructions following resection of primary bone tumors: a nationwide multicenter study". Journal of Bone and Joint Surgery American 96.4 (2014): e26.

65. Wu Z., et al. "Three-dimensional virtual bone bank system for selecting massive bone allograft in orthopaedic oncology". International Orthopaedics 39.6 (2015):1151-1158.

66. Gorbatov RO., et al. " [Precision personalized implants to replace bone defects for treatment of patients with osteoonkology]". Sovremennye Problemy Nauki I Obrazovaniya 6 (2016): 247.

67. Kang S., et al. "The MRI appearances of cancellous allograft bone chips after the excision of bone tumours". The Bone and Joint Journal 97-B.1 (2015): 121-128.

\section{Assets from publication with us}

- Prompt Acknowledgement after receiving the article

- Thorough Double blinded peer review

- Rapid Publication

- Issue of Publication Certificate

- High visibility of your Published work

Website: https://www.actascientific.com/

Submit Article: https://www.actascientific.com/submission.php Email us: editor@actascientific.com

Contact us: +919182824667 